\title{
CAPítulO 12
}

\section{GLUTAMATO MONOSSÓDICO ASPECTOS TOXICOLÓGICOS}

Felix Guillermo Reyes Reyes

Miguel Arcanjo Areas Hellen Dea Barros Maluly

\section{INTRODUÇÃ̃O}

O glutamato monossódico, também identificado pelas siglas GMS ou MSG (monosodium glutamate, em inglês), é o sal sódico do aminoácido ácido glutâmico, cujas estruturas estão apresentadas na Figura 12.1.

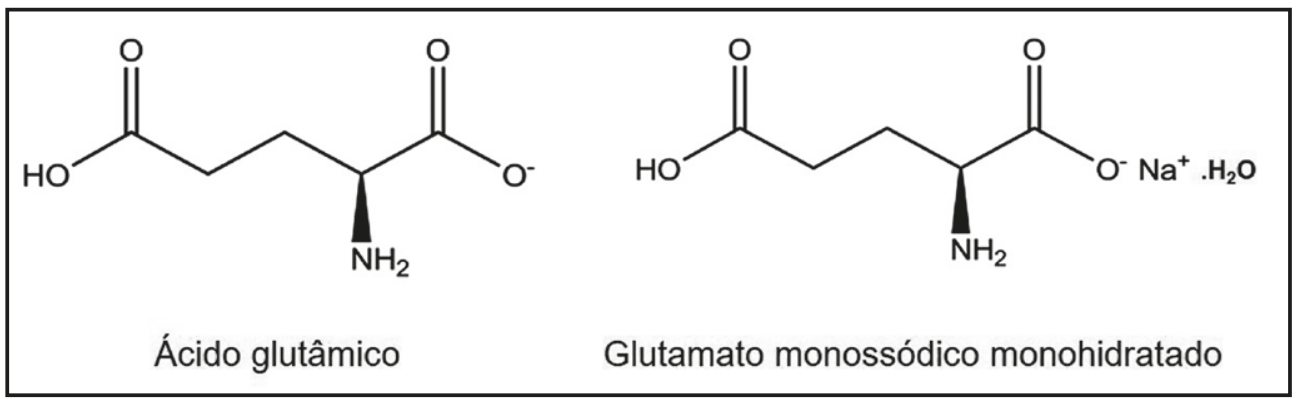

Figura 12.1 - Estruturas moleculares do ácido glutâmico e do glutamato monossódico mono-hidratado

Fonte: figura elaborada pelos autores. 
O ácido glutâmico (ou glutamato na sua forma dissociada) é o principal composto responsável pelo chamado quinto gosto básico ou gosto umami, palavra japonesa, que pode ser traduzida como "delicioso" ou "saboroso". Além do glutamato, outras moléculas podem induzir o gosto umami, tais como os nucleotídeos inosina-5'-monofosfato e guanosina-5'-monofosfato (Blonde \& Spector, 2017).

O glutamato livre, que não faz parte da estrutura de proteínas, pode estar naturalmente presente na composição de alimentos como, por exemplo, tomates, aspargos, milho, ervilha, peixes e frutos do mar e carnes em geral. Pode também estar presente em produtos processados e/ou fermentados como os queijos curados, presunto cru, salame ou ter sido adicionado, como aditivo alimentar na forma de MSG, em produtos processados como sopas prontas, molho de soja, molhos para salada e snacks, entre outros (Maluly et al., 2017; Yamaguchi \& Ninomiya, 2000).

O MSG tem sido alvo de pesquisas científicas em diversas áreas, tanto nas relacionadas à tecnologia de alimentos quanto às que envolvem a saúde humana. Esse composto tem um uso amplamente disseminado na indústria de alimentos, assim como desempenha muitas funções fisiológicas no corpo humano. Além disso, é importante mencionar que foi confirmada a presença de receptores específicos para o glutamato na língua, estômago e intestino (Kurihara, 2015).

Entretanto, a segurança do uso do MSG se tornou controversa a partir de publicações que implicavam em efeitos adversos à saúde humana, quando esse composto era adicionado a refeições ou produtos processados na forma de aditivo alimentar. Dentre os efeitos adversos podemos citar o "Complexo de Sintomas Relacionados à Ingestão de Glutamato Monossódico" (conhecido como "Síndrome do Restaurante Chinês") e "Obesidade Hipotalâmica" (Hermanussen et al., 2006; Kwok, 1968; Monno et al., 1995). Por esses motivos, a utilização do MSG como aditivo alimentar tem sido objeto de avaliações quanto a sua segurança de uso por parte de diferentes Comitês Científicos e/ou Agências de Regulamentação, como o Comitê Conjunto FAO/OMS de Especialistas em Aditivos Alimentares (JECFA), a Autoridade Europeia para Segurança de Alimentos (EFS), a Administração Federal de Alimentos e Medicamentos dos Estados Unidos da América (FDA) e a Federação das Sociedades Americanas para Biologia Experimental (FASEB).

Em 1970, na $14^{a}$ reunião, o JECFA avaliou diferentes sais do ácido glutâmico. O Comitê estabeleceu uma Ingestão Diária Aceitável (IDA) de 0-120 mg/kg p.c. (expressos como ácido L-glutâmico), concluindo ainda que essa IDA não se aplicava a crianças menores de 12 semanas de idade (JECFA, 1971). Entretanto, 
em 1973, na 17ª reunião, a restrição de uso para os alimentos infantis foi retirada, após a verificação de que os efeitos adversos não eram observados em animais neonatos nos níveis recomendados para o uso de sais do ácido glutâmico como aditivo alimentar, mantendo-se o valor de IDA (JECFA, 1974). No entanto, em avaliação posterior, realizada em 1987, o Comitê estabeleceu para o MSG uma IDA "não especificada". A denominação IDA "não especificada" significa que, tomando como base os dados disponíveis (químicos, bioquímicos, toxicológicos etc.), a ingestão diária total da substância, que se deriva de seu uso para alcançar os efeitos tecnológicos desejados e de sua concentração natural nos alimentos, não representa um risco para a saúde. Por esta razão, e pelas enunciadas em cada uma das avaliações, não se considerou necessário o estabelecimento de uma IDA expressa de forma numérica. Desta maneira, o JECFA concluiu que o MSG não apresenta risco à saúde, quando usado como aditivo alimentar (JECFA, 1988).

De forma similar ao JECFA, a Agência Nacional de Vigilância Sanitária do Brasil (ANVISA) também estabelece uma IDA "não especificada" para o MSG (BRASIL, 2001). Já nos Estados Unidos da América, em 1958, a FDA classificou o MSG como ingrediente Geralmente Reconhecido como Seguro (GRAS-Generally Recognized as Safe). Esta classificação foi mantida em reavaliação dos dados disponíveis sobre MSG, realizada em 1978 (FDA, 2018). Na década de 1980, trabalhos publicados indicaram a indução de efeitos adversos relacionados ao MSG como aditivo alimentar, principalmente em alimentos infantis (Airoldi et al., 1979; Arbogast \& Voogt, 1990). Consequentemente, a FDA recomendou que estudos adicionais fossem realizados para identificar a relação entre os efeitos adversos e o uso do MSG na alimentação de recém-nascidos e crianças (Anderson \& Raiten, 1992). Assim, a FASEB, que é um grupo de cientistas independentes, por solicitação da FDA, realizou uma revisão completa sobre os dados científicos relacionados à segurança do MSG, tendo concluído, em 1995, que o MSG é seguro quando consumido como aditivo alimentar nos níveis tecnológicos recomendados (0,1\%-0,8\% no alimento) (Beyreuther et al., 2007; Raiten et al., 1995).

O Parlamento Europeu publicou em 1995 uma diretiva que estabelece um limite de uso para o MSG de $10 \mathrm{~g} / \mathrm{kg}$ de alimento, quando utilizado como aditivo alimentar, individualmente ou em combinação com ácido glutâmico e seus sais (potássio, cálcio, amônia e magnésio), conforme as boas práticas de fabricação (EC, 1995). A última regulamentação publicada pelo parlamento foi em 2008, a qual manteve as recomendações da diretiva de 1995 (EC, 2008). Entretanto, em consequência da reavaliação da segurança de uso dos aditivos alimentares, 
o Setor sobre Aditivos Alimentares e Fontes de Nutrientes Adicionados aos Alimentos (ANS) da EFSA recomendou, em 2017, para o ácido glutâmico e seus sais uma IDA de grupo no valor de $30 \mathrm{mg} / \mathrm{kg}$ p.c., expressa como ácido glutâmico, o que não é compatível com o próprio consumo de glutamato intrínseco na dieta (Mortensen et al., 2017). Assim sendo, até 2020 essa recomendação não tinha sido aceita nem adotada pelos orgãos regulamentadores de todos os países.

Recentemente, Zanfirescu et al. (2019) relataram que muitos dos efeitos negativos para a saúde associados ao MSG têm pouca relevância em humanos, em relação à exposição crônica, e que são pouco informativos pois se baseiam em doses de exposição elevada que não atende aos níveis normalmente consumidos através dos alimentos. Os autores concluíram que estudos clínicos e epidemiológicos adicionais são necessários, com protocolo (desenho experimental) apropriado, levando em conta o MSG naturalmente presente nos alimentos e aquele adicionado como aditivo alimentar.

Este capítulo apresenta uma revisão dos dados biológicos, aspectos bioquímicos relacionados à cinética e metabolismo, assim como estudos especiais (transporte transplacentário, barreira hematoencefálica). Também são analisados os estudos de toxicidade (toxicidade aguda, subcrônica, crônica, teratogenicidade e carcinogenicidade), utilizados pelos diferentes Comitês e/ou Agências de Regulamentação para estabelecer o uso seguro do MSG como aditivo alimentar.

\section{ESTUDOS DE CINÉIICA E METABOLISMO}

\subsection{Comportamento do glutamato no trato gastrointestinal}

O glutamato é extensivamente metabolizado no trato gastrointestinal (Figura 12.2). O estudo de Reeds et al. (2000) demonstrou que no intestino de suínos [lactentes (até $20 \mathrm{~kg}$ ) e jovens (de 20 a $60 \mathrm{~kg}$ )], assim como em humanos adultos, o MSG ou glutamato da dieta é metabolizado em até $95 \%$ pelo efeito de primeira passagem. Posteriormente, o mesmo grupo de pesquisadores verificou que a absorção do glutamato no estômago é maior do que no intestino (Janeczko et al., 2007). 


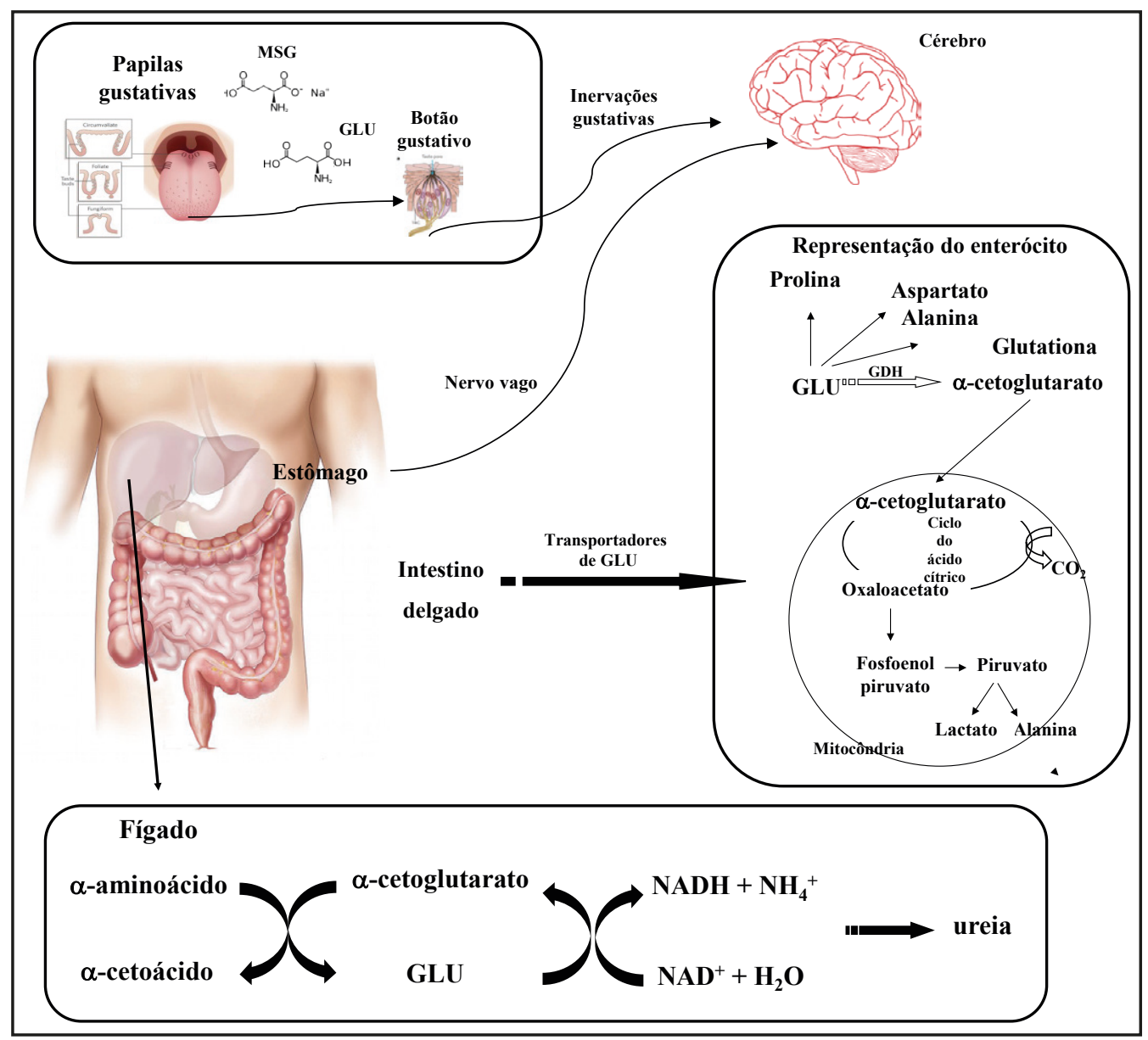

Figura 12.2 - Rota metabólica do MSG no organismo (MSG: glutamato monossódico; GLU: ácido glutâmico; GDH: glutamato desidrogenase; $\mathrm{CO}_{2}$ : dióxido de carbono; ATP: adenosina trifosfato; NAD: nicotinamida adenina dinucleotídeo; $\mathrm{NH}_{4}^{+}$: amônio)

Fonte: figura adaptada de Chandrashekar, 2006, e 3B Scientific/ https://www.3bscientific.com.br/index.html.

A metabolização do glutamato começa pelo seu transporte por parte das células do estômago e do intestino, através do transportador de glutamato-aspartato 1 (GLAST-1), do transportador do glutamato (GLT-1), dos carregadores de aminoácidos excitatórios 1 (EAAC-1) e dos transportadores de aminoácidos excitatórios 4 e 5. Os EAAC-1 são os transportadores mais abundantes de glutamato no intestino delgado, mas não no estômago e intestino grosso. Já o GLAST1 e o GLT-1 são expressos, na sua maior parte, no estômago. Quando o glutamato é absorvido pelas células do trato gastrointestinal, ele começa a ser catabolizado 
no citossol e na mitocôndria pela reação de transaminação, com atividade das enzimas aspartato aminotransferase, alanina aminotransferase, aminotransferases de cadeia ramificada e glutamato desidrogenase (GDH), presentes no estômago, intestino e colo. O glutamato é metabolizado a $\alpha$-cetoglutarato, o qual pode entrar no ciclo do ácido tricarboxílico com produção de energia (ATP) e liberação de dióxido de carbono (Iwanaga et al., 2005).

Os átomos de carbono do glutamato que não sofreram oxidação até a formação de dióxido de carbono são convertidos em lactato, alanina, prolina, citrulina, ornitina e arginina, que então entram na circulação portal. O nitrogênio derivado do metabolismo do glutamato é transformado em amônia e em outros aminoácidos, incluindo a citrulina, ornitina, prolina e arginina. Grande parte do nitrogênio destes compostos é convertida em ureia pelas células hepáticas, a qual é eliminada pela urina (Burrin \& Stoll, 2009).

Essa é a rota pela qual ocorre a metabolização do glutamato consumido normalmente pela população, incluindo o MSG utilizado como aditivo alimentar. Ainda, em um estudo realizado em suínos lactentes foi verificado que, mesmo em doses de MSG quatro vezes maiores daquelas normalmente utilizadas como aditivo alimentar (até 0,8\%), o glutamato livre não foi detectado no plasma (Haÿs et al., 2007).

\subsection{Metabolismo hepático do glutamato}

As rotas metabólicas do glutamato são complexas (Figura 12.2). Conforme mencionado anteriormente, o glutamato é extensivamente metabolizado no trato gastrointestinal em dióxido de carbono, lactato, glutationa, glutamina, alanina e vários outros tipos de aminoácidos. O glutamato ingerido, que não é utilizado no trato gastrointestinal, entra na circulação porta-hepática sendo, então, metabolizado no fígado. O esqueleto carbônico do glutamato pode ser oxidado através do ciclo de Krebs para gerar energia, e seu nitrogênio pode ser convertido em ureia, que é excretada na urina (Brosnan, 2000).

Sempre que o metabolismo dos aminoácidos é discutido, devem-se separar as vias relacionadas ao nitrogênio das vias dos esqueletos carbônicos. O metabolismo do nitrogênio é comum para muitos aminoácidos, porém, para o esqueleto carbônico geralmente é distinto. $\mathrm{O}$ fato mais importante do metabolismo do nitrogênio é a sua detoxificação na forma de ureia. O metabolismo de quase todos os aminoácidos se inicia pela ação de aminotransferases, sendo que o glutamato e o $\alpha$-cetoglutarato fazem parte dessa reação. A desaminação do glutamato promove a formação de $\alpha$-cetoglutarato e amônia, pela enzima glutamato desi- 
drogenase. A amônia produzida fornece um dos dois nitrogênios da ureia, pela carbamoil fosfato sintetase 1. A aspartato aminotransferase transfere o grupo amino do glutamato para oxaloacetato para produzir ácido aspártico, que pode então introduzir o segundo nitrogênio no ciclo da ureia pela argininosuccinato sintetase. Um dos aspectos desse processo é que as enzimas aminotransferase e glutamato desidrogenase (GDH) são reversíveis, o que permite que o fígado ajuste a produção de amônia e ácido aspártico, de acordo com as necessidades do ciclo da ureia. Assim, o glutamato além de produzir energia pela formação de $\alpha$-cetoglutarato, com posterior entrada no ciclo de Krebs, também possui um papel importante na regulação do ciclo da ureia (Brosnan \& Brosnan, 2009).

\subsection{Estudos da cinética}

As etapas da cinética do glutamato dependem da forma como ele se encontra no organismo (livre ou ligado às proteínas). Também há influência dos componentes da dieta, que dependem das concentrações de macronutrientes, principalmente de proteínas na dieta (Imada et al., 2014; Iwanaga et al., 2005; Luscombe-Marsh et al., 2009).

Em consequência do rápido metabolismo do glutamato livre nas células da mucosa intestinal e no fígado, os seus níveis plasmáticos sistêmicos são baixos, mesmo após a ingestão de elevadas quantidades de proteína na dieta. Todavia, a administração oral de doses elevadas de glutamato livre pode resultar na sua elevação nos níveis plasmáticos. Assim, o pico de concentração plasmática de glutamato irá depender da quantidade ingerida (Stegink et al., 1979). A administração, por gavagem, de uma dose de MSG de 1,0 g/kg p.c., em solução aquosa, resultou em um aumento significativo do glutamato plasmático em várias espécies estudadas. Os picos plasmáticos máximos de glutamato foram menores em macacos adultos e maiores em camundongos. Diferenças relacionadas à idade entre neonatos e adultos foram verificadas em camundongos e ratos, sendo que os picos plasmáticos e a área sob a curva foram maiores nos animais recém-nascidos do que nos adultos, pois recém-nascidos não possuem integridade gastrointestinal (Airoldi et al., 1979; Ohara \& Naim, 1977; Stegink et al., 1975).

Estudos sobre os efeitos dos componentes da dieta na absorção do glutamato têm sido realizados em animais de experimentação. Quando camundongos jovens receberam o MSG adicionado a fórmulas (alimentos) infantis, ou quando os humanos adultos receberam MSG com consomé através de intubação gástrica, os níveis máximos de glutamato no plasma foram significativamente menores, e o tempo para atingir o pico plasmático foi maior do que quando a mesma dose 
foi administrada com água (Ohara \& Naim, 1977; Stegink et al., 1985). De forma semelhante, em camundogos, a alimentação ad libitum de uma dieta contendo MSG, origina apenas ligeira elevação plasmática de glutamato acima dos níveis basais (Heywood et al., 1977).

Resultados similares da absorção de glutamato e dos níveis plasmáticos também foram verificados em humanos. Os dados mostraram apenas um leve aumento nos níveis plasmáticos de MSG, quando uma dose de $0,150 \mathrm{~g} / \mathrm{kg}$ p.c. foi ingerida com as refeições (Tung \& Tung, 1980). Como mencionado anteriormente, a ingestão de doses elevadas de MSG junto com refeições, provocou níveis plasmáticos de glutamato menores quando em comparação com água. Em geral, os alimentos que fornecem carboidratos metabolizáveis aumentam o metabolismo do glutamato e leva à redução dos seus níveis plasmáticos. Os carboidratos fornecem piruvato como substrato para transaminação com o glutamato nas células da mucosa, de modo que mais alanina é formada e menos glutamato atinge a circulação portal (Stegink et al., 1983). Cabe mencionar, ainda, que crianças, inclusive bebês prematuros, têm a mesma capacidade de metabolizar doses semelhantes de MSG administradas em fórmula infantil (Tung \& Tung, 1980).

A FASEB revisou trinta e quatro estudos realizados em roedores, onde o MSG foi administrado por via enteral, sendo que em quatorze o MSG foi administrado por gavagem ou intubação intragástrica, treze na dieta ou água, quatro por ambos os métodos e em três, não foi especificada a rota de administração (Anderson \& Raiten, 1992). Em nove estudos, as concentrações de ácido glutâmico no plasma foram determinadas e em cinco foram avaliados os picos de concentração plasmática (dois em ratos e três em camundongos). Os pesquisadores concluíram que as lesões produzidas no núcleo arqueado do hipotálamo, quando o MSG era administrado por via enteral, foram devido ao fato dos estudos terem sido realizados em animais jovens, cuja barreira hematoencefálica está em formação, e com doses elevadas, o que não reflete o consumo como aditivo alimentar. A menor dose em que algum tipo de lesão foi observada foi de $0,5 \mathrm{~g} /$ kg p.c. em animais com oito dias de idade (Daabees et al., 1985).

\section{ESTUDOS DE TOXICIDADE}

\subsection{Exposição aguda por via oral}

Os ensaios de toxicidade aguda visam demonstrar a ocorrência de efeito adverso em curto período de tempo. Geralmente, os ensaios tratam da administração de uma dose única ou exposições múltiplas em 24 h. Considera-se também 
o aparecimento de um efeito num período de até 14 dias, após a administração da substância estudada (Barlow et al., 2002). Estudos para a determinação do valor da dose letal mediana $\left(\mathrm{DL}_{50}\right)$, nos quais o $\mathrm{MSG}$ foi administrado por via oral, foram realizados em diferentes espécies sendo, na sua maioria, publicados nas décadas de 1960 e 1970 (JECFA, 1988). Esses trabalhos mostraram valores variáveis de $\mathrm{DL}_{50}$ em camundongos (13-19,2 g/kg p.c.), em ratos (10-19,9 g/kg p.c.), e em coelhos (níveis maiores que $2,3 \mathrm{~g} / \mathrm{kg}$ p.c.) (JECFA, 1988). Esses dados demonstram que os valores de $\mathrm{DL}_{50}$ são muito elevados indicando que o MSG possui baixo potencial de toxicidade aguda, e também não correspondem aos níveis de consumo de MSG, quando utilizado como aditivo alimentar.

Os efeitos tóxicos tais como lesão no núcleo arqueado do hipotálamo e alterações hipotalâmicas, com consequências neuroendócrinas em roedores neonatos, têm sido associados à exposição aguda ao MSG. Esses efeitos ocorreram após administração de doses elevadas por via parenteral. Verificaram-se também efeitos neuronais, após algumas horas da exposição, quando o MSG foi administrado por via oral, em altas doses, em camundongos neonatos, sem a presença de alimentos. No entanto, em outras espécies, nenhum efeito adverso foi observado (Dawson, 1983; Hermanussen et al., 2006; Olney, 1971).

\subsection{Exposição subcrônica (curta duração), por via oral}

A toxicidade subcrônica (de curta duração) geralmente envolve o estudo de efeitos adversos decorrentes da exposição a múltiplas doses do agente tóxico, durante períodos que não excedem $10 \%$ da vida média do animal, com o objetivo de obter informações que revelem possíveis efeitos tóxicos, em um período suficiente em que não haja modificações relativas à idade, como alterações morfológicas e funcionais dos tecidos (Barlow et al., 2002).

Doses elevadas de MSG, administradas por via enteral, podem desenvolver lesões em duas áreas do cérebro (hipotálamo e hipocampo) que são particularmente suscetíveis ao glutamato, quando este promove efeitos excitatórios exacerbados. Para verificar esses efeitos, ratos adultos foram submetidos à administração oral de doses relativamente elevadas de MSG (4,0 g/kg p.c.). Foi avaliada a ingestão única de MSG por gavagem e de doses repetidas durante 21 dias, nas quais o MSG foi administrado na dieta. Os níveis de glutamato no plasma e no cérebro também foram determinados. Foram realizadas análises histológicas de tecidos cerebrais para avaliar a ocorrência de possíveis alterações neuropatológicas. No estudo de ingestão aguda, verificou-se um aumento significativo de glutamato no plasma e nos níveis extracelulares no hipocampo 
e no hipotálamo, em comparação os ratos do grupo controle. Entretanto, nenhuma alteração nos níveis de glutamato foi verificada no estudo de 21 dias em comparação aos ratos que receberam dieta controle. A avaliação histológica não revelou nenhuma alteração associada à administração do MSG, tanto aguda como subcrônica (Monno et al., 1995).

Na reavaliação da segurança de uso do MSG realizada pela ANS/EFSA (Mortensen et al., 2017) foram considerados estudos de curta duração (28 dias) realizados em ratos de ambos os sexos (fêmeas doses de 4,8 e 4,9 g/ $\mathrm{kg}$ p.c. e machos doses de 5,1 e 5,3 g/kg p.c.), não tendo sido observados efeitos adversos. Em cachorros beagle (doses de 0,15, 0,5 e $5 \mathrm{~g} / \mathrm{kg}$ p.c. administradas pela dieta) foram observados efeitos clínicos, tais como vômito e diarreia, porém não foram considerados relevantes ou foram apenas transitórios. Assim, lesões inespecíficas, que não foram consideradas como efeitos adversos, ocorreram no peso do timo de fêmeas que foram submetidas às doses mais elevadas. Ensaios subcrônicos realizados em cães foram realizados para verificar possíveis efeitos adversos no trato urinário, foram relatados apenas efeitos como aumento do volume urinário e secreção de sódio, após 2 anos de estudo. Este efeito foi observado na dose de administração mais elevada de MSG (2,5 g/kg p.c.).

\subsection{Toxicidade crônica (longo prazo) e carcinogenicidade}

Os estudos de toxicidade crônica e carninogenicidade são realizados num período correspondente à vida média do animal. O principal objetivo dos estudos é avaliar os mecanismos para possíveis lesões neoplásicas consequentes da exposição à substância, em várias doses, por uma rota apropriada de administração (para aditivos, via oral) (Barlow et al., 2002).

Owen et al. (1978) relataram que a ingestão de MSG causava hiperplasia de bexiga e da pélvis renal em ratos. Posteriormente foi verificado que esse efeito não era provocado pela ingestão de MSG e sim devido à presença de bicarbonato de potássio $\left(\mathrm{KHCO}_{3}\right)$ na dieta administrada aos animais, o qual tinha um efeito alcalinizante na urina ( $\mathrm{pH}$ aumentava até 8), durante o período de maior consumo de alimento (de Groot et al., 1988).

Shibata et al. (1995), realizaram ensaio em ratos, os quais foram alimentados com dietas contendo $0,0,6,1,25,2,5$ e 5,0\% de MSG, por um período de 2 anos. Os animais que receberam dieta com 5,0\% de MSG tendiam ao retardo do crescimento. Ocorreu aumento do $\mathrm{pH}$ e do sódio urinário e diminuição do potássio em ratos de ambos os sexos que receberam dieta com 2,5 e 5,0\%. Todavia, nenhum desenvolvimento de lesão proliferativa ou neoplásica foi observado no trato urinário. 


\subsection{Estudos de reprodução e teratogenicidade}

Nos estudos de reprodução e teratogenecidade são avaliados os efeitos adversos da substância no sistema de reprodução de animais de experimentação e as possíveis consequências na sua prole. Assim, ensaios para avaliação dos efeitos adversos do MSG sobre o sistema reprodutivo de animais de experimentação e suas possíveis consequências na sua prole. Foram utilizadas doses de MSG de 2,5 e 4,0 g/kg p.c., por via oral, em camundongos na fase final de gestação. Foi avaliado o comportamento da mãe e da prole. O acasalamento dos machos tratados com as fêmeas tratadas resultou em gestações e filhos normais, indicando que a administração de MSG não afeta a capacidade reprodutiva da prole numa fase tardia da gravidez (Yu et al., 1997).

Estudos de reprodução e teratogenicidade com administração de MSG por via oral foram avaliados pelo JECFA (JECFA, 1988). A monografia relata que os animais expostos não apresentaram efeitos adversos, nem mesmo quando as fêmeas eram alimentadas com altas doses de glutamato, indicando que o feto e o neonato (lactentes) não foram expostos a níveis tóxicos pela dieta materna, por transferência transplacentária ou pelo leite das lactantes. Esse fato está de acordo com os relatos de que os níveis de glutamato no sangue fetal não aumentam paralelamente com o aumento dos níveis de glutamato no sangue materno. Por exemplo, as ratas que receberam, por via oral, doses de 8,0 g/ $\mathrm{kg}$ p.c. ao final do período de gestação, tiveram aumento dos níveis plasmáticos de 100 para $1.650 \mathrm{nmol} / \mathrm{mL}$, porém não houve aumento significativo nos níveis plasmáticos dos fetos. Do mesmo modo, em fêmeas prenhas de macacos Rhesus, a infusão de $1,0 \mathrm{~g}$ de MSG/h levou a um aumento de 10 a 20 vezes nos níveis de glutamato no plasma materno, mas não ocorreu nenhuma mudança nos níveis plasmáticos dos fetos. Também em ratos e macacos, a ingestão oral de elevadas doses de MSG não levou à detecção de qualquer aumento nos níveis do ácido glutâmico no leite materno.

Outros estudos avaliaram a passagem de diferentes aminoácidos através da placenta de ovinos e concluíram que o glutamato é o aminoácido que possui menor passagem para circulação uterina, pois é metabolizado em $\alpha$-cetoglutarato na própria parede uterina (Cetin, 2001; Holzman et al., 1979).

\subsection{Mutagenicidade}

$\mathrm{Na}$ década de 1970, foram realizados estudos para avaliar o potencial de mutagenicidade do MSG. Assim, células de tecidos de ratos foram expostas a 
uma dose de $0,1 \%$ de MSG, em solução, e observadas durante 72 h. Nenhum efeito tóxico foi observado (FDA, 1969). Outro estudo utilizou camundongos machos e administrou MSG por gavagem em diferentes níveis (0, 2,7 e 5,4 g/ $\mathrm{kg}$ p.c.). Os animais tratados acasalaram com fêmeas não tratadas a cada seis semanas consecutivas. As fêmeas foram sacrificadas na metade da gravidez e o útero foi examinado para avaliação de sinais de morte embrionária precoce e efeitos mutagênicos. Não foram verificadas diferenças com relação à implantação, reabsorção e deficiência embrionária, entre as fêmeas que acasalaram com animais tratados e não tratados (JECFA, 1988).

\subsection{Neurotoxicidade}

Efeitos neurotóxicos associados à exposição ao MSG foram inicialmente relatados nas décadas de 1960 e 1970 . Os principais relatos sobre as lesões neuronais relacionadas à administração de MSG foram realizados por Olney (1971), que administrou doses de 0,5-4,0 g/kg p.c. de MSG, por via parenteral, em camundongos com 2 a 9 dias de idade. Os animais foram sacrificados decorridos 30 minutos ou 48 h após a administração da substância. Foram observadas lesões no núcleo arqueado do hipotálamo. As mesmas lesões também foram detectadas após a administração de 5,0-7,0 g/ kg p.c. em camundongos adultos.

Outros pesquisadores também têm utilizado como modelo o animal tratado com doses elevadas de MSG, administradas por via parenteral ou oral. Os estudos indicaram que a elevação dos níveis de glutamato no plasma podem aumentar a circulação de glutamato no cérebro e provocar lesão no núcleo arqueado do hipotálamo e, como consequência, obesidade e diabetes (Hermanussen et al., 2006; Nagata et al., 2006). Nesses experimentos, a barreira hematoencefálica de roedores foi lesionada de forma focada. Em animais que tinham recebido uma injeção contínua de MSG, o conteúdo de água no cérebro aumentou de forma significativa, provocando edema. A duplicação da concentração de glutamato no plasma foi suficiente para causar este efeito, no qual o edema cerebral piorou apenas nestes animais. Provavelmente, o edema pode ter ocorrido pela absorção de glutamato nas células da glia, o que reduz o glutamato extracelular juntamente com íons sódio, forçando a entrada de água no cérebro.

Verificou-se também que as concentrações de glutamato no plasma, em condições normais, podem chegar a $100 \mu \mathrm{mol} / \mathrm{L}$ e no cérebro até $12.000 \mu \mathrm{mol} / \mathrm{L}$, mas apenas $0,5-2,0 \mu \mathrm{mol} / \mathrm{L}$ chega até o fluido extracelular. Além disso, a barreira hematoencefálica é quase impermeável à passagem de glutamato, mesmo em altas concentrações, exceto em algumas áreas pequenas com capilares 
fenestrados, pois o glutamato é um composto polar e, portanto, o influxo passivo é limitado a menos de $1 \%$ daquele que ocorre nas veias sanguíneas de outros tecidos (Hawkins, 2009).

\section{0 MSG E A SíNDROME DO RESTAURANTE CHINÊS}

A Síndrome do Restaurante Chinês (ou Complexo de Sintomas relacionados ao MSG) foi descrita primeiramente por Kwok, em 1968, que relatou um conjunto de sinais e sintomas, como por exemplo, dores no pescoço ou na cabeça, fraqueza e palpitações. Além disso, outros sintomas têm sido relacionados à ingestão de MSG, como asma, dermatite atópica, urticária, dificuldades respiratórias e taquicardia, através do consumo de comida chinesa, ou mais precisamente, através de alimentos que continham MSG (Allen et al., 1987; Ratner et al., 1984; Van Bever et al., 1989).

Geha et al. (2000) realizaram um estudo sobre essa síndrome utilizando um desenho experimental do tipo duplo-cego, placebo-controlado. O estudo avaliou frequência cardíaca, pressão sanguínea, taxa respiratória e temperatura para verificar as respostas que eram consideradas como positivas a reações adversas descritas após o consumo de MSG (fraqueza geral, tensão e contração muscular, rubor, suor, sensação de queimação, dor de cabeça, enxaqueca, dor no peito, palpitações, dormência e formigamento). Os resultados eram considerados positivos se o indivíduo apresentava 2 ou mais desses sintomas. O desenho experimental consistiu em quatro ensaios sequenciais (A-D), sendo que os dois primeiros foram idênticos a um estudo realizado no Canadá, em 1997 (Yang et al., 1997). Nos ensaios A-C, o MSG foi administrado sem alimentos. $\mathrm{O}$ ensaio A foi realizado com indivíduos $(\mathrm{n}=130)$ que diziam ter reações após o consumo de MSG. Os participantes receberam cápsulas de placebo ou cápsulas com 5,0 g de MSG em dias alternados. Tanto placebo como MSG foram administrados junto com líquido com sabor cítrico para evitar que sentissem o sabor da substância do teste. As reações foram registradas durante $2 \mathrm{~h}$. Os indivíduos considerados positivos passaram para o ensaio B, cujo objetivo foi analisar se as respostas dos indivíduos eram consistentes e se eram dependentes da dose. Para isso, várias concentrações de MSG [0 (placebo), 1,25, 2,5 ou 5 g]/200 mL da mesma solução cítrica foram administradas em dias diferentes. Os resultados mostraram que os sintomas apareceram com mais frequência conforme a dose de MSG aumentava. Os indivíduos que responderam a $5 \mathrm{mg}$ de MSG e não ao placebo nos ensaios A e B $(n=12)$ passaram para o ensaio $C$, cujo objetivo foi confirmar a reprodutibilidade dos resultados obtidos nos experi- 
mentos anteriores. Apenas 2 indivíduos responderam a $5 \mathrm{~g}$ de MSG, mas não ao placebo, os quais foram escolhidos para o ensaio D. O protocolo do ensaio D consistiu na avaliação das características clínicas das reações ao MSG versus placebo quando ingeridos com alimentos. Assim, os 2 indivíduos ingeriram placebo (3 vezes) ou $5 \mathrm{~g}$ de MSG (3 vezes) na presença de alimentos (leite e cereais). Ambos os indivíduos responderam a apenas 1 das 3 administrações de MSG. Além disso, os sintomas descritos foram diferentes dos relatados nos ensaios anteriores (A-C). Geha et al. (2000) concluíram que quando um grupo de indivíduos autoidentificados sensíveis ao MSG é solicitado a mostrar reprodutibilidade dos diferentes sintomas que eles mesmos relatavam, nenhum consegue fazê-lo.

Nas suas conclusões da avaliação sobre a segurança do MSG, a FASEB e FDA não consideraram a existência de uma subpopulação sensível e concordaram com a avaliação de segurança do JECFA e do Comitê Científico para Alimentos da Comissão da Comunidade Europeia (SCF) (Walker \& Lupien, 2000).

Williams \& Woessner (2009) realizaram uma revisão da literatura disponível sobre a provável participação do MSG em reações alérgicas mais comuns (asma, urticária e rinite), que fazem parte do "complexo de sintomas atribuídos ao MSG". Os pesquisadores concluíram que, no caso específico da asma, a análise crítica dos métodos e das propostas experimentais apresentam limitações que impossibilitam concluir a existência dessa relação. Além disso, estudos com maior rigor científico (como aqueles que envolvem testes duplo-cego-placebo-controlado) demonstraram ser improvável que o consumo de MSG tenha papel participativo na asma, mesmo nos indivíduos identificados como sensíveis a esta substância.

Em contrapartida, muitos estudos que examinaram o provável papel da ingestão alimentar de MSG como agente causador de urticária (edema geralmente pruriginoso) e angioedema (inchaço localizado na derme ou submucosa), sugeriram a possibilidade de um caso raro, que provavelmente representa menos de 3\% dos casos já relatados na literatura (Bush \& Montalbano, 2014). Entretanto, a qualidade da evidência que sustenta essa relação está longe da ideal. A evidência de que o MSG é agente causador de rinite está limitada a dois relatos na literatura científica. Resumindo, é essencial mencionar que a evidência científica atual não indica que o MSG participe do aparecimento de asma, urticária, angioedema ou rinite (Williams \& Woessner, 2009). 


\section{CONSIDERAÇÕES FINAIS}

O MSG é um sal do ácido glutâmico e é utilizado mundialmente como aditivo alimentar pela indústria alimentícia, com a finalidade de realçar o sabor e auxiliar na redução de sódio em alimentos. Todos os sais deste aminoácido se dissociam em solução aquosa. Portanto, o glutamato presente nas soluções é o mesmo glutamato livre presente naturalmente nos alimentos (como queijos, carnes, tomates etc.).

No corpo humano, o glutamato proveniente da dieta, tanto na sua forma natural (encontrado nos alimentos) quanto adicionado como aditivo alimentar é, na sua maior parte, metabolizado no próprio trato gastrointestinal. O glutamato possui toxicidade aguda muito baixa. Quando administrado por via oral, a $\mathrm{DL}_{50}$ (dose letal para 50\% dos animais testados) em ratos e camundongos é de, aproximadamente, $13-19 \mathrm{~g} / \mathrm{kg}$ p.c.

Estudos de toxicidade subcrônica e crônica, com duração de até dois anos em ratos e camundongos, incluindo a fase reprodutiva, não revelaram nenhum efeito adverso específico quando o MSG foi administrado na dieta em até 4,0\%. Pesquisas de dois anos em cães, com 10\% de MSG na dieta, não revelaram nenhum efeito no ganho de peso, peso dos órgãos, alterações clínicas, mortalidade ou comportamento em geral. Na avaliação de segurança realizada pelo JECFA, o Comitê concluiu que a ingestão dietética total de sais de ácido glutâmico, decorrentes do seu uso como aditivo alimentar em níveis necessários para atingir o efeito tecnológico desejado e de sua aceitabilidade em alimentos, não representa um risco para a saúde. Portanto, a expressão de um valor numérico para IDA não foi considerada necessária e, assim, foi atribuído para sais de ácido glutâmico (sais de monossódio, potássio, cálcio e amônio) uma IDA "não especificada". O JECFA também verificou que não haveria necessidade de restrições para mulheres grávidas e crianças, mas mantiveram a posição colocada nas outras avaliações realizadas, que aditivos alimentares, em geral, não devem ser consumidos por neonatos, antes de doze semanas de idade.

O SCF, em 1991, chegou à mesma conclusão do JECFA, atribuindo para sais de ácido glutâmico uma IDA "não especificada" (Walker \& Lupien, 2000). Todavia, em 1995, uma diretiva da Comunidade Europeia (EC, 95) sobre aditivos alimentares fixou um limite de uso de $10 \mathrm{~g} / \mathrm{kg}$ para L-glutamato, individualmente ou em combinação com ácido glutâmico e seus e sais presentes nos produtos alimentícios, com exceção de alimentos não processados, alimentos para bebês (para os quais, o uso do glutamato e seus sais não é permitido) e temperos e 
especiarias, fato que foi mantido na Resolução 1.333/2008 (EC, 2008). Além disso, em 2017, como resultado da reavaliação do uso seguro do glutamato e seus sais, como aditivos alimentares, a ANS/EFSA recomendou uma IDA de grupo de $30 \mathrm{mg} / \mathrm{kg}$ p.c., expressa como ácido glutâmico (Mortensen et al., 2017).

A FASEB publicou, em 1995, a avaliação realizada sobre reações adversas ao MSG e concluiu que, embora haja evidências científicas comprovadas de efeitos adversos em alguns indivíduos sensíveis a doses elevadas de glutamato, não há documentação suficiente para indicar que existe um subgrupo de indivíduos saudáveis que apresentam reações adversas ao MSG. O subgrupo que responde às manifestações do conjunto de sintomas relacionados ao MSG, geralmente responde dentro de 1 hora de exposição, quando exposto a uma dose oral de 3,0 g de MSG na ausência de alimento (Anderson \& Raiten, 1992).

O FDA adotou as conclusões da FASEB com relação ao complexo de sintomas, salientando que há diferenças em testes onde o MSG é administrado em cápsulas ou em soluções, na ausência ou na presença de alimentos. O FDA também concluiu que não há evidência de que o glutamato livre, presente naturalmente em alimentos ou adicionado a dieta, cause danos degenerativos a células nervosas em longo prazo. O FDA também considerou as conclusões do relatório da FASEB coerentes com as avaliações de segurança feitas por outras organizações competentes (incluindo o JECFA e SCF), que afirmaram a segurança do MSG nos níveis normalmente consumidos pela população em geral. Assim, o FDA considera o MSG como um ingrediente alimentar, como o sal e o açúcar. Tal como esses ingredientes, o MSG também não pode ser usado em excesso, pois seu uso é autolimitante. Se utilizado em excesso, não melhora o sabor dos alimentos e, ao contrário, o piora (Beyreuther et al., 2007; Jinap \& Hajeb, 2010).

Assim, considerando todas as avaliações realizadas por comitês científicos, órgãos e agências de regulamentação, pode-se concluir que o uso do MSG como aditivo alimentar é seguro, se utilizado na quantidade necessária para obter efeito tecnológico desejado e conforme as boas práticas de produção de alimentos.

\section{REFERÊNCIAS BIBLIOGRÁFICAS}

AIROLDI, L. et al. "Glutamic acid and sodium levels in the nucleus arcuatus of the hypothalamus of adult and infant rats after oral monosodium glutamate". Toxicology Letters. 3(3): 121-126, 1979. 
ALLEN, D. H.; DELOHERY, J. \& BAKER, G. "Monosodium L-glutamate-induced asthma". The Journal of Allergy and Clinical Immunology. 80(4): 530537, 1987.

ANDERSON, S. A. \& RAITEN, D. J. (ed.). Safety of amino acids used as dietary supplements. Bethesda, FASEB, 1992. Disponível em https://www.faseb. org/Portals/2/PDFs/LSRO_Legacy_Reports/1992_Safety Amino Acids Used As Dietary Supplmnts.pdf. Acesso em 15/1/2020.

ARBOGAST, L. A. \& VOOGT, J. L. "Sex-related alterations in hypothalamic tyrosine hydroxylase after neonatal monosodium glutamate treatment". Neuroendocrinology. 52(5): 460-467, 1990.

BARLOW, S. et al. "Hazard identification by methods of animal-based toxicology". Food and Chemical Toxicology. 40(2-3): 145-191, 2002.

BEYREUTHER, K. et al. "Consensus meeting: monosodium glutamate - an update”. European Journal of Clinical Nutrition. 61(3): 304-313, 2007.

BLONDE, G. D. \& SPECTOR, A. C. "An examination of the role of L-glutamate and inosine 5'-monophosphate in hedonic taste-guided behavior by mice lacking the T1R1 + T1R3 receptor". Chemical Senses. 42(5): 393-404, 2017.

BRASIL. "Resolução - RDC n.1, de 2 de janeiro de 2001. A Agência Nacional de Vigilância Sanitária aprova o regulamento técnico que aprova o uso de aditivos com a função de realçadores de sabor, estabelecendo seus limites máximos para os alimentos". Diário Oficial da União. 2001. Disponível em http://portal.anvisa.gov.br/documents/33916/391619/Resolucao_RDC_n1_ de_02_de_janeiro_de_2001.pdf/f3ce5586-b054-4a0a-8762-10d7db6d1789. Acesso em 2/2/2020.

BROSNAN, J. T. "Glutamate, at the interface between amino acid and carbohydrate metabolism”. The Journal of Nutrition. 130(4): 988S-990S, 2000.

BROSNAN, M. E. \& BROSNAN, J. T. "Hepatic glutamate metabolism: a tale of 2 hepatocytes". The American Journal of Clinical Nutrition. 90(3): 857S-861S, 2009.

BURRIN, D. G. \& STOLL, B. "Metabolic fate and function of dietary glutamate in the gut". The American Journal of Clinical Nutrition. 90(3): 850S-856S, 2009. 
BUSH, R. K. \& MONTALBANO, M. "Asthma and food additives. Chapter 27". In: METCALFE, D. D. et al. (ed.). Food allergy: adverse reactions to foods and food additives. Fifth Edition, Chichester, John Wiley \& Sons Ltd., 2014, pp. 341-345.

CETIN, I. "Amino acid interconversions in the fetal-placental unit: the animal model and human studies in vivo". Pediatric Research. 49(2): 148-154, 2001.

CHANDRASHEKAR, J. et al. "The receptors and cells for mammalian taste". Nature. 444 (7117): 288-294, 2006.

DAABEES, T. T. et al. "Correlation of glutamate plus aspartate dose, plasma amino acid concentration and neuronal necrosis in infant mice". Food and Chemical Toxicology. 23(10): 887-893, 1985.

DAWSON, R. "Acute and long lasting neurochemical effects of monosodium glutamate administration to mice". Neuropharmacology. 22(12A): 1417-1419, 1983.

DE GROOT, A. P.; FERON, V. J. \& IMMEL, H. R. "Induction of hyperplasia in the bladder epithelium of rats by a dietary excess of acid or base: implications for toxicity/carcinogenicity testing". Food and Chemical Toxicology. 26(5): 425-434, 1988.

EC. "European Parliament and Council Directive No 95/2/EC of 20 February 1995 on food additives other than colours and sweeteners". 1995. Disponível em https://eur-lex.europa.eu/LexUriServ/LexUriServ.do?uri=CONSLEG:1995L0002:20060815:EN:PDF. Acesso em 2/2/2020.

EC. "Regulation (EC) No 1333/2008 of the European Parliament and of the Council of 16 December 2008 on food additives. 2008”. Disponível em https:// eur-lex.europa.eu/legal-content/EN/TXT/PDF/?uri=CELEX:32008R1333\&from $=E N$. Acesso em 2/2/2020.

FDA, Food and Drug Administration. "Report on monosodium glutamate for review by Food Protection Committee, NAS/NRC”. In: Apud - JECFA - Joint FAO/WHO Expert Committee on Food Additives (1988). "L-glutamic acid and its ammonium, calcium, monosodium and potassium salts". WHO - World Health Organization (ed.). 1969. Disponível em http://www.inchem.org/documents/jecfa/jecmono/v22je12.htm. Acesso em 2/2/2020. 
FDA, Food and Drug Administration. "GRAS Substances (SCOGS) Database". 2018. Disponível em https://www.fda.gov/food/ingredientspackaginglabeling/ gras/scogs/default.htm. Acesso em 2/2/2020.

GEHA, R. S. et al. "Multicenter, double-blind, placebo-controlled, multiple-challenge evaluation of reported reactions to monosodium glutamate". Journal of Allergy and Clinical Immunology. 106(5): 973-980, 2000.

HAWKINS, R. A. "The blood-brain barrier and glutamate". The American Journal of Clinical Nutrition. 90(3): 867S-874S, 2009.

HAŸS, S. P. et al. "Dietary glutamate is almost entirely removed in its first pass through the splanchnic bed in premature infants". Pediatric Research. 62(3): 353-356, 2007.

HERMANUSSEN, M. et al. "Obesity, voracity and short stature: the impact of glutamate on the regulation of appetite". European Journal of Clinical Nutrition. 60(1): 25-31, 2006.

HEYWOOD, R.; JAMES, R. W. \& WORDEN, A. N. "The ad libitum feeding of monosodium glutamate to weanling mice". Toxicol. Lett. 1(3): 151-155, 1977.

HOLZMAN, I. R. et al. "Uterine uptake of amino acids and placental glutamine--glutamate balance in the pregnant ewe". Journal of Developmental Physiology. 1(2): 137-149, 1979.

IMADA, T. et al. "Supplementing chicken broth with monosodium glutamate reduces energy intake from high fat and sweet snacks in middle-aged healthy women". Appetite. 79: 158-165, 2014.

IWANAGA, T.; GOTO, M. \& WATANABE, M. "Cellular distribution of glutamate transporters in the gastrointestinal tract of mice: an immunohistochemical and in situ hybridization approach". Biomedical Research. 26(6): 271-278, 2005.

JANECZKO, M. J. et al. "Extensive gut metabolism limits the intestinal absorption of excessive supplemental dietary glutamate loads in infant pigs". The Journal of Nutrition. 137(11): 2384-2390, 2007.

JECFA, Joint FAO/WHO Expert Committee on Food Additives. "Evaluation of food additives: specifications for the identity and purity of food additives and their toxicological evaluation: some extraction solvents and certain other substances; and a review of the technological efficacy of some antimicrobial 
agents". Fourteenth Report of the Joint FAO/WHO Expert Committee on Food Additives. Geneva, 1971. Disponível em https://apps.who.int/iris/bitstream/handle/10665/40848/WHO_TRS_462.pdf;jsessionid=8A7F9685E9E54180C3AFE5B97D88676E?sequence=1. Acesso em 2/2/2020.

JECFA, Joint FAO/WHO Expert Committee on Food Additives. "Toxicological evaluation of certain food additives with a review of general principles and of specifications". Seventeenth report of the Joint FAO/WHO Expert Committee on Food Additives. Geneva, 1974. Disponível em https://apps.who.int/iris/ bitstream/handle/10665/41072/WHO_TRS_539.pdf?sequence=1. Acesso em 2/2/2020.

JECFA, Joint FAO/WHO Expert Committee on Food Additives. "L-glutanic acid and its ammonium, calcium, monosodium and potassium salts". In: WHO - World Health Organization (ed.). 22nd ed. New York, Cambridge University Press, 1988. pp. 97-161. Disponível em http://www.inchem.org/documents/ jecfa/jecmono/v22je01.htm. Acesso em 2/2/2020.

JINAP, S. \& HAJEB, P. “Glutamate. Its applications in food and contribution to health". Appetite. 55(1): 1-10, 2010.

KURIHARA, K. "Umami the fifth basic taste: history of studies on receptor mechanisms and role as a food flavor". BioMed Research International. 2015:189402, 2015.

KWOK, R. H. "Chinese-restaurant syndrome". The New England Journal of Medicine. 278(14): 796, 1968.

LUSCOMBE-MARSH, N. D.; SMEETS, A. J. P. G. \& WESTERTERP-PLANTENGA, M. S. "The addition of monosodium glutamate and inosine monophosphate-5 to high-protein meals: effects on satiety, and energy and macronutrient intakes”. British Journal of Nutrition. 102(06): 929, 2009.

MALULY, H. D. B.; ARISSETO-BRAGOTTO, A. P. \& REYES, F. G. R. “Monosodium glutamate as a tool to reduce sodium in foodstuffs: Technological and safety aspects". Food Sci. Nutr. 5(6): 1039-1048, 2017.

MONNO, A. et al. "Extracellular glutamate levels in the hypothalamus and hippocampus of rats after acute or chronic oral intake of monosodium glutamate". Neuroscience Letters. 193(1): 45-48, 1995. 
MORTENSEN, A. et al. "Re-evaluation of glutamic acid (E 620), sodium glutamate (E 621), potassium glutamate (E 622), calcium glutamate (E 623), ammonium glutamate (E 624) and magnesium glutamate (E 625) as food additives". EFSA Journal. 15(7), 2017.

NAGATA, M. et al. "Type 2 diabetes mellitus in obese mouse model induced by monosodium glutamate”. Experimental Animals. 55(2): 109-115, 2006.

OHARA, I. \& NAIM, M. "Effects of monosodium glutamate on eating and drinking behavior in rats". Physiology \& Behavior. 19(5): 627-634, 1977.

OLNEY, J. W. "Glutamate-induced neuronal necrosis in the infant mouse hypothalamus. An electron microscopic study". Journal of Neuropathology and Experimental Neurology. 30(1): 75-90, 1971.

OWEN, G. et al. "The feeding of diets containing up to $4 \%$ monosodium glutamate to rats for 2 years". Toxicology Letters. 1: 221-226, 1978.

RAITEN, D. J.; TALBOT, J. M. \& FISHER, K. D. (ed.). “Executive summary from the report: analysis of adverse reactions to monosodium glutamate (MSG)". The Journal of Nutrition. 125(11): 2891S-2906S, 1995. Disponível em https:// doi.org/10.1093/jn/125.11.2891S. Acesso em 26/3/2020.

RATNER, D.; ESHEL, E. \& SHOSHANI, E. "Adverse effects of monosodium glutamate: a diagnostic problem". Israel Journal of Medical Sciences. 20(3): 252-253, 1984.

REEDS, P. J. et al. "Intestinal glutamate metabolism". The Journal of Nutrition. 130(4): 978S-982S, 2000.

SHIBATA, M. A. et al. "Lack of carcinogenicity of monosodium 1-glutamate in Fischer 344 rats". Food and Chemical Toxicology. 33(5): 383-391, 1995.

STEGINK, L. D. et al. "Comparative metabolism of glutamate in the mouse, monkey and man". In: FILER, L. J. et al. (ed.). Glutamic acid: Advances in Biochemistry and Physiology. New York, Raven Press, 1979, pp. 5-102.

STEGINK, L. D. et al. "Monosodium glutamate metabolism in the neonatal monkey”. American Journal of Physiology-Legacy Content. 229(1): 246-250, 1975.

STEGINK, L. D.; FILER, L. J. \& BAKER, G. L. "Plasma amino acid concentrations in normal adults fed meals with added monosodium L-glutamate and aspartame". The Journal of Nutrition. 113(9): 1851-1860, 1983. 
STEGINK, L. D.; FILER, L. J. \& BAKER, G. L. "Plasma glutamate concentrations in adult subjects ingesting monosodium L-glutamate in consommé". The American Journal of Clinical Nutrition. 42(2): 220-225, 1985.

TUNG, T.C. \& TUNG, K. S. "Serum free amino acid levels after oral glutamate intake in infant and adult humans". Nutr Rep Int. 22: 431-443, 1980.

VAN BEVER, H. P.; DOCX, M. \& STEVENS, W. J. "Food and food additives in severe atopic dermatitis". Allergy. 44(8): 588-594, 1989.

WALKER, R. \& LUPIEN, J. R. "The safety evaluation of monosodium glutamate”. The Journal of Nutrition. 130(4): 1049S-1052S, 2000.

WILLIAMS, A. N. \& WOESSNER, K. M. 'Monosodium glutamate 'allergy': menace or myth?”. Clinical \& Experimental Allergy. 39(5): 640-646, 2009.

YAMAGUCHI, S. \& NINOMIYA, K. "Umami and food palatability". The Journal of Nutrition. 130(4S Suppl): 921S-926, 2000.

YANG, W. H. et al. "The monosodium glutamate symptom complex: assessment in a double-blind, placebo-controlled, randomized study". The Journal of Allergy and Clinical Immunology. 99(6 Pt 1): 757-762, 1997.

YU, T. et al. "Effects of maternal oral administration of monosodium glutamate at a late stage of pregnancy on developing mouse fetal brain". Brain Research. 747(2): 195-206, 1997.

ZANFIRESCU, A. et al. "A review of the alleged health hazards of monosodium glutamate". Comprehensive Reviews in Food Science and Food Safety. 18(4): 1111-1134, 2019. 